\title{
Evaluation and management of the difficult airway : Fiberoptic intubation in a patient with glottic tumor
}

\author{
Y. H. Hsu, Y. T. Chang \\ Taichung Veterans General Hospital, Department of Anesthesiology, Taichung, Taiwan
}

\section{Background}

Performing fiberoptic intubation in a nearly obstructed airway has always been a big challenge for anesthesiologists.

\section{Case Report}

A sixty year-old man suffered from dyspnea and expiratory stridor for one week. The man had dysphonia and needed oxygen supplement while supine. Sagittal, axial and coronal views of the computed tomography showed a tumor (figure1) nearly obstructing the glottic opening. Tumor biopsy and protective tracheostomy were planned. We measured the diameter of the airway in the CT scan and presumed the 5.0-mm ID endotracheal tube (ETT) should pass through. After light sedation with midazolam and analgesia with xylocaine spray $10 \%$ in his supraglottic airway, we performed oral fiberoptic intubation while the otolaryngologist scrubbed up for emergent tracheostomy. Bronchoscopy showed a rounded tumor nearly covering up the vocal cord. A gap between the tumor and glottic opening appeared during inspiration that allowed the doctor to pass through the 3.1-mm OD bronchoscope and gently advance the ETT over the scope.

\section{Discussion}

Difficult airway management is often unpredictable. Although many devices exist to assist with intubation, being able to evaluate the anatomy thoroughly under more accurate guidance, such as CT scan, would be safer for patients and make doctors more confident while manipulating the airway. Unfortunately, anesthesiologists may have to deal with the gap between the static preoperative image and the dynamic degree of airway obstruction. We should always be cautious and keep the second plan handy, such as fully-prepared surgeon for emergent tracheostomy, if the first one failed.
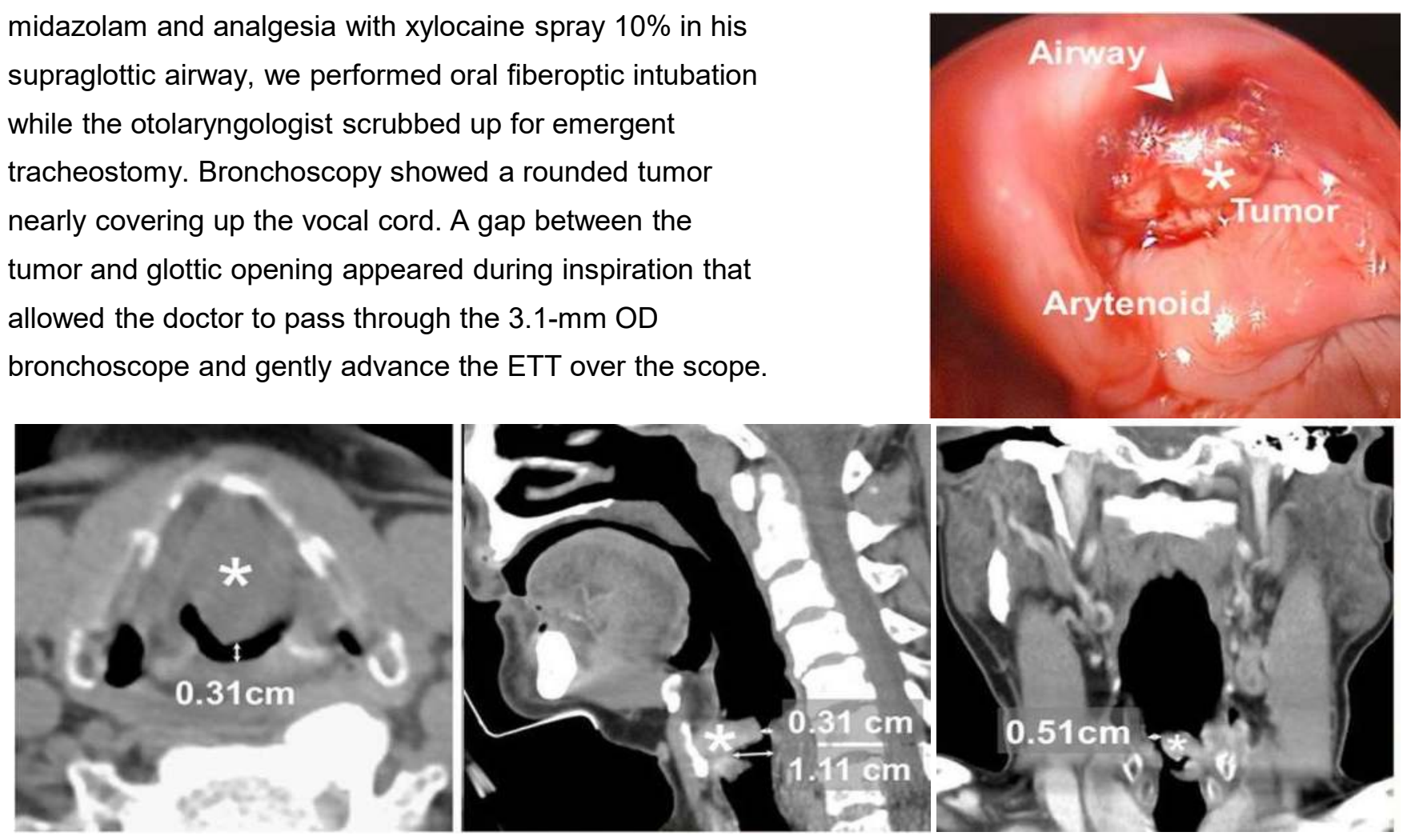

\section{Learning points}

This case highlights the importance of preoperative evaluation and coordination with the surgeon. In the future, we may perform fiberoptic examination preoperatively in order to evaluate the severity of airway obstruction in real-time and come up with a more precise plan ahead. 